\title{
Investigation of geometrical defect of AA6061-T4 cold embossing using FEM
}

\begin{abstract}
Accuracy of the net-shape manufacturing such as forging becomes critical as the process depends on many factors. Defect will harm the assembly tend to affect the performance of the part. Therefore, it must be detected and minimized it as soon as the manufacturing begins. Small size and complexity of the part limits the defective part to be assessed. The paper presents an investigation on the defect of the cold embossing pin. In this work, the effect of design and process parameters to the formation of defect was studied using 2-D FE analysis. The defect can be measured based on the incomplete filling of the region from the FE result. The results show that, the FE observations are in good agreement with the experimental result.
\end{abstract}

Keyword: Assembly accuracy; Cold embossing; Filling ratio; Forging defect; Image processing 